\title{
Banco de dentes humanos no Brasil: revisão de literatura
}

\author{
Dayliz Quinto Pereira*
}

\author{
* Departamento de Saúde. Universidade Estadual de Feira de \\ Santana, Bahia. Doutorando em Medicina e Saúde. Faculdade de \\ Medicina da Bahia. Universidade Federal da Bahia
}

\section{RESUMO}

O presente artigo descreve resultados de uma revisão de literatura sobre "Banco de Dentes Humanos no Brasil - BDH", com o objetivo de conhecer a produção científica sobre $\mathrm{BDH}$ no país, a sua relação com o ensino, a pesquisa, a extensão, a sua estrutura administrativa e as suas implicações legais, tendo como base a Bioética. A busca teve como base de dados a Biblioteca Virtual em Saúde de Odontologia e livros especializados. Foram encontrados oitenta e um artigos e dois livros, selecionando-se vinte e três artigos e dois livros, um sobre Banco de Dentes $\mathrm{Hu}-$ manos e outro Serviços Odontológicos da ANVISA. Os textos analisados foram classificados em três categorias: Ensino/Pesquisa, Organização/Estrutura dos BDH e Bioética/Biossegurança. Conclui-se que poucos são os trabalhos relacionados aos $\mathrm{BDH}$ nos cursos de Odontologia no Brasil, o que demonstra a necessidade de novas reflexões na Odontologia sob o olhar da Bioética na criação dos BDH.

\section{DESCRITORES}

Banco de Dentes. Biossegurança. Bioética. Cursos de Odontologia.

A criação dos Bancos de Dentes Humanos (BDH) nas instituições de ensino superior no Brasil teve inicio por volta do ano de 2000 com o objetivo de minimizar o comércio ilegal de estruturas dentárias, assim como desenvolver uma percepção dos discentes e profissionais da área de Odontologia acerca da Biossegurança, das questões legais e das discussões em Bioética.

O BDH é responsável pelas atividades de recepção, preparação, desinfecção, manipulação, seleção, preservação, catalogação, estocagem, cessão, empréstimo, administração dos dentes doados e educação para a ética.

Os dentes humanos extraídos são frequentemente utilizados no ensino odontológico de Anatomia e de Histologia, assim como, na prática em laboratório pré-clínica e em pesquisas científicas da graduação e pós-graduação.

Assim, o ensino odontológico necessita de grande número de unidades dentárias. Todavia, "muitos acadêmicos e profissionais obtêm, junto a outros profissionais tais como os coveiros de cemitérios, dentes cujas doações não estão sendo registradas conforme as determinações legais". ${ }^{1}$

O objetivo deste estudo é conhecer a produção cientifica sobre BDH no Brasil, a sua relação com o ensino, a pesquisa, a sua estrutura administrativa e as suas implicações legais, tendo como base a Bioética.

\section{METODOLOGIA}

A revisão da produção científica existente teve como base livros especializados, teses, dissertações e periódicos indexados no banco de dados da Biblioteca Virtual em Saúde (BVS) através do formulário de pesquisa avançada do Portal de Revistas Científicas em Ciências da Saúde:

- Bibliografia Brasileira de Odontologia (BBO),

- U.S. National Library of Medicine (PubMed/ MEDLINE),

- Scientific Library online (SciELO),

- Literatura Latino-Americana e do Caribe em Ciências da Saúde (LILACS).

O período da coleta compreendeu os anos de 2008 a 2012.

A metodologia aplicada foi "resultante da reunião e análise dos trabalhos referentes ao tema objeto da pesquisa", com a intenção de "identificar na literatura o referencial teórico do estudo" e cujos autores já se ocupavam do tema até o momento da pesquisa. $^{2}$

Como estratégia de busca usaram-se critérios de inclusão através das palavras: Banco de dentes humanos, dentes, ética, bioética, doação de órgãos, transplante dental, educação em Odontologia, biossegu- 
rança. Durante a busca utilizou-se os operadores boleanos and e or, e textos na língua inglesa, francesa, italiano e em espanhol.

Os critérios de exclusão estão relacionados ao conteúdo dos artigos, no momento da leitura dos respectivos resumos ou abstracts. Após leitura, foram retirados os textos que não se relacionavam com o tema e trabalhos não indexados na base de dados. Procurou-se selecionar os artigos, respeitando os conteúdos próprios do tema sobre "Banco de Dentes Humanos".

Após seleção do material, iniciou-se a leitura mais apurada, através de uma análise de conteúdo, direcionando os tópicos do texto para o objeto de estudo.

\section{RESULTADOS}

No período de 2008 a 2012, foram identificados 81 artigos, sendo excluídos 64 artigos que não se relacionavam diretamente com o objeto do estudo, restando 23 artigos. Destes, treze no Lilacs e BVS igualmente, e dez no BBO. Na busca por outras fontes foram selecionados dois livros, nos quais um o tema é abordado como principal, e o outro um capítulo do livro.

Nos Quadros 1 e 2 os artigos em número de dezessete e seis respectivamente encontram-se organizados por ordem cronológica conforme título, autoria, ano e periódico. Nos dois livros em separado, além dos itens de referência incluiu-se editora.

Com base neste levantamento bibliográfico e analisando os textos, os temas foram classificados em três categorias:

1. Ensino/Pesquisa

2. Organização/Estrutura dos BDH

3. Bioética/Biossegurança

\section{DISCUSSÃO}

\section{Ensino/Pesquisa}

Os textos mencionam a importância dos $\mathrm{BDH}$ para os cursos de graduação e pós-graduação em Odontologia, ao mesmo tempo em que, não dissociam a responsabilidade do envolvimento com a Ética.

Para a aprendizagem dos discentes de graduação em Odontologia tanto na teoria como na prática, as unidades dentárias são elementos fundamentais para o ensino e a pesquisa, o que é reforçada pela opinião dos Prof. Vanzelli e Imparato ${ }^{3}$ e em 2003 no Livro de "Banco de Dentes Humanos"4 e em outros textos: "A utilização de dentes humanos para fins de pesquisa ou realização de procedimentos laboratoriais e clínicos deve respeitar aspectos éticos e legais, e precisa ser uma preocupação de pesquisadores, educadores, alunos e da população em geral."

Em outro artigo, ${ }^{5}$ "em relação à solicitação de elementos dentais para a utilização na graduação, o resultado da pesquisa foi unânime, visto que $100 \%$ dos alunos, do primeiro ao quinto ano, necessitam de dentes extraídos para o curso. Em relação à obtenção dos elementos dentais, $84,2 \%$ dos alunos relataram dificuldade para a aquisição dos dentes solicitados nas disciplinas do curso". No ensino odontológico é fundamental a "utilização de dentes humanos (...) para o aprendizado do aluno de Odontologia, que é obrigado, em quase todas as faculdades, a 'arrumar' o material. Alguns professores nem questionam como o aluno adquiriu os dentes, que são geralmente comprados em clínicas particulares, cemitérios ou com colegas já formados". ${ }^{6}$

Calculando matematicamente, um curso de graduação em Odontologia com 30 alunos por semestre/ano precisaria de 840 unidades dentárias para o ensino a partir das aulas de Anatomia, seguidas de Dentística, Endodontia e outras disciplinas. Portanto, um curso de Odontologia com 60 alunos/ano matriculados necessitará de 1.680 unidades dentárias por ano e um estoque permanente de 3.360 dentes, o que torna muitas vezes difícil a captação, a distribuição e controle no $\mathrm{BDH}$ para os discentes e professores.

Portanto a utilização das unidades dentárias nos cursos de graduação é uma necessidade e uma realidade tanto no ensino odontológico como na pesquisa odontológica.

Um levantamento sobre o uso de dentes na pesquisa apresentado na $17^{\mathrm{a}}$ e $18^{\mathrm{a}}$ Reuniões Anuais da Sociedade Brasileira de Pesquisa Odontológica ( $\mathrm{SBPqO}$ ) "demonstrou que, de 2.569 trabalhos apresentados, $834(32,5 \%)$ utilizaram dentes naturais, resultando em média de 34 dentes por pesquisa". ${ }^{7}$ Ainda no mesmo artigo encontramos o cálculo do Prof. Imparato da Universidade de São Paulo, "para o número de dentes utilizados nos cursos de Odontologia: uma faculdade gasta de três a quatro mil dentes por semestre". ${ }^{7}$ Portanto, após o mapeamento dos BDH no Brasil em 2011 identificou-se 196 cursos de graduação em Odontologia, sendo necessário aproximadamente $600 \mathrm{mil} \mathrm{a}$ 800 mil dentes para as atividades acadêmicas.

Outro estudo ${ }^{8}$ de 2010 apresentou a utilização de dentes extraídos, humanos ou não, "nas pesquisas publicadas a partir de 1996, em periódicos brasileiros de acesso online gratuito e com informações da origem dos dentes. Dos quatorzes periódicos publicados, foram utilizados 8.921 dentes humanos e 2.920 não humanos". 
Quadro 1 - Artigos e livros nacionais selecionados para a revisão de literatura, segundo título, autor, ano, tipo de publicação no período de 2001-2011.

\begin{tabular}{|c|c|c|c|c|}
\hline № & Título & Autor & Ano & Periódico \\
\hline 1 & $\begin{array}{l}\text { Estágio atual da organização dos bancos de dentes } \\
\text { humanos nas faculdades de odontologia do território } \\
\text { brasileiro }\end{array}$ & $\begin{array}{l}\text { Begosso M, Imparato J, } \\
\text { Duarte D }\end{array}$ & 2001 & $\begin{array}{l}\text { - Revista de Pós-Graduação } \\
\text { da USP } \\
\text { - ISSN 0104-5695 }\end{array}$ \\
\hline 2 & Comercialização de dentes nas universidades & $\begin{array}{l}\text { Paula S, Bittencourt LP, } \\
\text { Pimentel E, Gabrielli Filho PA, } \\
\text { Imparato JCP }\end{array}$ & 2001 & $\begin{array}{l}\text { - Revista Pesquisa Brasileira } \\
\text { de Odontopediatria e } \\
\text { Clinica Integrada } \\
\text { - ISSN 1519-0501 }\end{array}$ \\
\hline 3 & Estruturação de um banco de dentes humanos & $\begin{array}{l}\text { Nassif A, Tieri F, Ana P, Botta S, } \\
\text { Imparato JCP }\end{array}$ & 2003 & $\begin{array}{l}\text { - Pesquisa Odontológica } \\
\text { Brasileira } \\
\text { - ISSN 1517-7491 }\end{array}$ \\
\hline 4 & Banco de dentes: uma idéia promissora & Vanzelli M, Imparato JCP & 2003 & $\begin{array}{l}\text { - Stomatos } \\
\text { - ISSN 1519-4442 }\end{array}$ \\
\hline 5 & $\begin{array}{l}\text { Banco de dentes: ética e legalidade no ensino, } \\
\text { pesquisa e tratamento odontológico }\end{array}$ & $\begin{array}{l}\text { Ferreira EL, Fariniuk LF, } \\
\text { Cavali AÉC, Baratto Filho F, } \\
\text { Ambrósio AR }\end{array}$ & 2003 & $\begin{array}{l}\text { - Revista Brasileira de } \\
\text { Odontologia } \\
\text { - ISSN 1984-3747 }\end{array}$ \\
\hline 6 & A bioética na odontologia & Pires LAG, Cerveira J & 2003 & $\begin{array}{l}\text { - Stomatos } \\
\text { - ISSN 1519-4442 }\end{array}$ \\
\hline 7 & $\begin{array}{l}\text { Conhecimento dos alunos do curso de odontologia da } \\
\text { USS sobre banco de dentes humanos }\end{array}$ & $\begin{array}{l}\text { Rabello TB, Souza MCA, Silva } \\
\text { FSP, Madruga FF }\end{array}$ & 2005 & $\begin{array}{l}\text { - Revista Brasileira de } \\
\text { Odontologia } \\
\text { - ISSN 1984-3747 }\end{array}$ \\
\hline 8 & $\begin{array}{l}\text { Estruturação do banco de dentes humanos decíduos } \\
\text { da Universidade Federal de Santa Maria/ RS/ Brasil }\end{array}$ & $\begin{array}{l}\text { Marin E, Zorzin D, Mainardi AP, } \\
\text { Oliveira MDM }\end{array}$ & 2005 & $\begin{array}{l}\text { - Revista de Odontologia de } \\
\text { Passo Fundo } \\
\text { - ISSN 1413-4012 }\end{array}$ \\
\hline 9 & $\begin{array}{l}\text { Banco de dentes humanos numa instituição de ensino: } \\
\text { importância, implementação e funcionamento (revisão } \\
\text { de literatura) }\end{array}$ & Melo CRO & 2005 & $\begin{array}{l}\text { - Monografia apresentada na } \\
\text { ABO /MG }\end{array}$ \\
\hline 10 & $\begin{array}{l}\text { Dentes humanos no ensino odontológico: procedência, } \\
\text { utilização, descontaminação e armazenamento pelos } \\
\text { acadêmicos da UNIMONTES }\end{array}$ & $\begin{array}{l}\text { Costa SM, Mameluque S, } \\
\text { Brandão EL, Melo AEMA, Pires } \\
\text { CPAB, Rezende EJC, Alves KM }\end{array}$ & 2005 & $\begin{array}{l}\text { - Revista da ABENO } \\
\text { - ISSN 1679-5954 }\end{array}$ \\
\hline 11 & $\begin{array}{l}\text { Avaliação do nível de conhecimento dos acadêmicos } \\
\text { do curso de odontologia da UNIVILLE sobre a utilização } \\
\text { de dentes extraídos na graduação e banco de dentes }\end{array}$ & $\begin{array}{l}\text { Zucco D, Kobe R, Fabre C, } \\
\text { Madeira L, Baratto Filho F }\end{array}$ & 2006 & $\begin{array}{l}\text { - Revista Sul Brasileira de } \\
\text { Odontologia } \\
\text { - ISSN 1806-7727 }\end{array}$ \\
\hline 12 & $\begin{array}{l}\text { Percepção de acadêmicos de odontologia sobre } \\
\text { clonagem, doação de órgãos e banco de dentes. }\end{array}$ & $\begin{array}{l}\text { Garbin CAS, Garbin AJI, } \\
\text { Santos KTS, Pacheco AC }\end{array}$ & 2008 & $\begin{array}{l}\text { - Revista de Pós-Graduação } \\
\text { da USP } \\
\text { - ISSN 0104-5695 }\end{array}$ \\
\hline 13 & $\begin{array}{l}\text { Banco de dentes humanos para o ensino e pesquisa } \\
\text { odontológica }\end{array}$ & $\begin{array}{l}\text { Moreira L, Genari B, Stello R, } \\
\text { Collares FM, Samuel SMW }\end{array}$ & 2009 & $\begin{array}{l}\text { - Rev. Faculdade Odontologia } \\
\text { Porto Alegre } \\
\text { - ISSN 0566-1854 }\end{array}$ \\
\hline 14 & $\begin{array}{l}\text { Conhecimento popular, acadêmico e profissional sobre } \\
\text { o banco de dentes humanos }\end{array}$ & $\begin{array}{l}\text { Pinto SL, Silva SP, Barros LM, } \\
\text { Tavares EP, Silva JBOR, } \\
\text { Freitas ABDA }\end{array}$ & 2009 & $\begin{array}{l}\text { - Revista Brasileira de } \\
\text { Odontopediatria e Clinica } \\
\text { Integrada } \\
\text { - ISSN 1519-0501 }\end{array}$ \\
\hline 15 & $\begin{array}{l}\text { Uso de dentes extraídos nas pesquisas odontológicas } \\
\text { publicadas em periódicos brasileiros de acesso online } \\
\text { gratuito: um estudo sob o prisma da bioética }\end{array}$ & $\begin{array}{l}\text { Freitas ABDA, Castro CDL, Sett } \\
\text { GS, Barros LM, Moreira AN, } \\
\text { Magalhães CS }\end{array}$ & 2010 & $\begin{array}{l}\text { - Arquivos em Odontologia } \\
\text { - ISSN 1516-0939 }\end{array}$ \\
\hline 16 & $\begin{array}{l}\text { Banco de dentes humanos na percepção dos } \\
\text { acadêmicos da Faculdade de Odontologia da } \\
\text { Universidade Federal Fluminense }\end{array}$ & $\begin{array}{l}\text { Maggioni AR, Scelza MFZ, } \\
\text { Silva LE, Salgado VE, Borges } \\
\text { DO, Maciel ACC }\end{array}$ & 2010 & $\begin{array}{l}\text { - Revista Fluminense de } \\
\text { Odontologia } \\
\text { - ISSN 1413-2966 }\end{array}$ \\
\hline 17 & $\begin{array}{l}\text { Banco de dentes humanos no curso de odontologia da } \\
\text { ULBRA - Campos Torres }\end{array}$ & $\begin{array}{l}\text { Vinholes JIA, Fernandes DC, } \\
\text { Ritzel IF }\end{array}$ & 2011 & $\begin{array}{l}\text { - Conversas Interdisciplinares } \\
\text { - ISSN 2176-1051 }\end{array}$ \\
\hline 1 & Banco de dentes humanos & Imparato JCP e cols. & 2003 & $\begin{array}{l}\text { - Editora Maio } \\
\text { - ISSN 85-877543-2-7 }\end{array}$ \\
\hline 2 & $\begin{array}{l}\text { Manual de prevenção e controle de riscos em serviços } \\
\text { odontológicos - O órgão dental e a importância dos } \\
\text { bancos de dentes }\end{array}$ & Silva TR, Ferreira EL & 2006 & $\begin{array}{l}\text { - Capítulo do Livro da } \\
\text { ANVISA } \\
\text { - ISSN 84-334-1050-6 }\end{array}$ \\
\hline
\end{tabular}

Fonte: presente pesquisa. 
Quadro 2 - Artigos Internacionais selecionados para a revisão de literatura, segundo título, autor, ano, tipo de publicação no período de 1968 - 2011.

\begin{tabular}{|c|l|l|l|l|}
\hline No & \multicolumn{1}{|c|}{ Título } & \multicolumn{1}{c|}{ Autor } & \multicolumn{1}{c|}{ Ano } & \multicolumn{1}{c|}{ Periódico } \\
\hline 1 & Editorial prospect on tooth bank & Coburn RJ & 1968 & Abstract Dental \\
\hline 2 & La banca del dente & Muratori G & 1969 & Dental Cadmos \\
\hline 3 & Les implants biologiques et “la banque des dents” & $\begin{array}{l}\text { chercheve M, Hubert JP, } \\
\text { Bordon R }\end{array}$ & 1969 & Promot Dent \\
\hline 4 & $\begin{array}{l}\text { Les transplantations dentaires: constitution d'une } \\
\text { banque de dents et problèmes de stérilisation }\end{array}$ & $\begin{array}{l}\text { mathieu L, Fleurette J, } \\
\text { Transy MJ }\end{array}$ & 1970 & $\begin{array}{l}\text { Ann Odontostomatol } \\
\text { (Lyon) }\end{array}$ \\
\hline 5 & Come organizzare una banca del dente & muratori G & 1976 & Dental Cadmos \\
\hline 6 & Banche dei denti & Muratori G & 1986 & Dental Cadmos \\
\hline
\end{tabular}

Fonte: presente pesquisa.

O BDH é importante não só no armazenamento das unidades dentárias, mas sim, promover a sensibilização dos indivíduos sobre o elemento dentário, como órgão e suscitar discussões sobre o tema relativas a questões da Bioética e da Biossegurança.

\section{Organização/Estrutura dos BDH}

Em 1969 o cirurgião-dentista italiano Muratori no $\operatorname{artigo}^{9}$ "La Banca Del Dente" cita o então cirurgião-dentista francês Joseph Jean François Lemaire pela sua técnica de transplante dentário, como são adquiridas as unidades dentárias e a sua forma de conservação.

"Ele desenvolveu com sucesso em NY onde transplanta 123 dentes e com este sucesso passa a oferecer dois guinéus por dente anterior das pessoas que estavam dispostas a vender seus elementos dentários". Talvez sua intenção, era criar uma espécie de Banco de dentes (BD) antes do seu tempo, para ter sempre os dentes disponíveis para transplante. (texto traduzido na integra) (...) Só que o BD do Lemaire resultou em um fiasco solene por várias razões, especialmente para os muito (...) que se alegram em tratar dentes extraídos (falta de higiene, a ausência, naqueles dias, dos antibióticos).$^{9}$

Assim, a atual iniciativa do banco de dente não pode ser comparada à antiga, mencioná-la apenas por curiosidade. $\mathrm{O}$ "banco permite ao dentista ter a sua disposição, material biológico para transplante".

Pesquisadores franceses no artigo" 10 "Les Implants Biologiques et La Banque des dents" descrevem que os resultados em longo prazo foram tão satisfatórios que criam um BD como uma associação de praticantes (sem fins lucrativos). Conferidos os interessados podem enviar-nos os dentes extraídos há menos de 15 dias e que foram armazenados imediatamente após avulsão em solução salina, sem qualquer trata- mento prévio. (...) No mesmo texto solicitam aos cirurgiões-dentistas e aos estomatologistas que enviem dentes para um endereço e assim adquirem pleno direito tornar-se membro associado do BD (...) Em seu retorno do laboratório, eles estão prontos para transplante. Isto lhes permite manter quase indefinidamente. "Acreditamos que essa conquista, sem duvida, abre novos horizontes para a profissão". ${ }^{10}$

Os textos apresentam as primeiras noções de BDH onde a prioridade são os implantes dentários, a confecção de próteses, ao mesmo tempo em que oferecem "guinéus por dente anterior das pessoas que estavam dispostas a vender seus elementos dentários" " para a manutenção do "banco permitindo ao dentista ter a sua disposição, material biológico para transplante" , pode-se pressupor o inicio do $\mathrm{BDH}$ e do comércio das unidades dentárias.

No Brasil a primeira idéia de Banco de Dentes surge por volta de 1981, quando Garbrielli et al. ${ }^{11}$ "criaram o método de colagem em dentes anteriores, no qual a seleção de um dente para restabelecer a fratura coronária (...) foi através de um Banco de Dentes".

Os autores ${ }^{12-14}$ são unânimes em afirmar a importância do BDH nos cursos de Odontologia, em relação ao funcionamento, organização e estrutura física, discorrem sobre a responsabilidade na rotina de arrecadação, preparação, descontaminação, armazenamento e distribuição. Assim como, "estimular as doações, ao mesmo tempo em que, conscientiza a população e a comunidade científica sobre a valorização do dente como órgão". ${ }^{4}$

Segundo o Manual de prevenção e controle de riscos em serviços odontológicos, ${ }^{1}$ "para o funcionamento de um banco de dentes são necessários alguns requisitos como, infraestrutura adequada, equipamentos próprios e a contratação de pessoal técnico especializado e auxiliar, bem como o estabelecimen- 
to de fluxos e rotinas próprias que norteiem todas as etapas referentes à captação e distribuição dos órgãos dentários".

Nos artigos selecionados ${ }^{5,12,14-17}$ a estrutura física descrita apresenta-se superficialmente, quando no livro sobre Banco de Dentes na p.55 cita que "a infraestrutura oferecida a cada um dos $\mathrm{BDH}$ poderá variar de uma faculdade para outra, mas a sua organização é fundamental para o bom funcionamento e sua característica como um Banco de Dentes Humanos".

Ainda sobre a organização e infraestrutura outro artigo sugere que "para realização das funções do $\mathrm{BDH}$, é necessário um laboratório e uma sala de suporte. O laboratório deverá ser construído de acordo com as normas da vigilância sanitária correntes." ${ }^{18}$

Portanto os artigos ${ }^{3,4,7,12,15,19,20}$ relatam que se deve respeitar as normas de biossegurança e da vigilância sanitária preconizada pela ANVISA para os ambientes similares, mas não especificam detalhadamente como deve ser a infraestrutura do BDH nas Instituições de Ensino Superior, o que gera algumas dúvidas, com relação à sala de suporte das clínicas e laboratórios de práticas odontológicas.

Em relação à captação das unidades dentárias existem várias formas, uma delas acontece na própria clínica ou ambulatório do curso de Odontologia, onde graduandos, professores e pesquisadores, prestam assistência à população em geral.

"O BDH deverá responsabilizar-se pela obtenção de uma quantidade de dentes que seja necessária para a demanda das instituições as quais o Banco de dentes auxilia. As fontes de arrecadação podem ser as mais variadas: clínicas particulares, postos de saúde, clínicas da própria faculdade ou instituição de ensino, hospitais"18 e além destas a "doação espontânea das coleções de dentes particulares tanto dos profissionais quanto dos acadêmicos para o pleno funcionamento dos bancos". ${ }^{21}$

Outro ponto importante e que os autores 5,12,13,15,20,22 consideram "preocupante" é o risco de infecção cruzada com a manipulação de material biológico advindo dos dentes, ao mesmo tempo em que "enfatizam o aspecto de ainda não se ter encontrado um método de esterilização ou uma solução desinfetante que não interfira (...) nas propriedades físico-químicas dos dentes (...)"18

\section{Bioética/Biossegurança}

Os pesquisadores ${ }^{3,4,23}$ afirmam que a "valorização do dente é um fato pouco considerado pela maioria dos odontólogos e por alguns profissionais vinculados à pesquisa científica", quando "utilizam grandes quantidades de dentes humanos, em seus trabalhos, desconsiderando os aspectos éticos e legais" que dizem respeito à origem destes órgãos.

"Em 1997, com a formulação da Lei de Transplante no Brasil, os dentes passaram a ser reconhecidos como órgãos. Sendo assim, torna-se necessária a autorização do doador para a utilização de dentes". ${ }^{7}$

Alguns aspectos depois desta lei sobre a valorização atribuída aos dentes, "dizem respeito à polpa dentária que passa a ser também estudada para possíveis doadores de células-tronco, junto como células do cérebro, dos olhos, na pele, nos músculo. Entretanto, ainda não se sabe se essas células residem nestes tecidos ou se originam de células tronco hematopoiéticas circulantes. Daí a necessidade de intensificar as discussões sobre a clonagem, como a doação de órgãos, principalmente a doação do órgão dental e a criação dos Bancos de Dentes". ${ }^{17}$

Outro aspecto importante que deve ser considerado pelos profissionais de Odontologia, diz respeito às mutilações dentárias "adquiridas pelo homem, por meio de falhas continuadas na conduta clínica profissional ou por problemas que há tempos acompanham às questões de saúde pública no Brasil, os quais, muitas vezes, inviabilizam o acesso da população aos serviços de saúde odontológicos". ${ }^{17}$

Por outro lado, "a Odontologia como ciência, ensina que a avulsão de um elemento dentário pode acarretar danos funcionais, estéticos e fonéticos. $\mathrm{O}$ dente é um órgão complexo e importante para a saúde do indivíduo, de tal maneira que não pode ser substituído de forma totalmente satisfatória por qualquer tipo de reparação protética”. ${ }^{24}$

Sabe-se que outra forma de aquisição das unidades dentárias baseia-se no comércio ilegal de dentes que é frequente, e principalmente no meio universitário, "em estudo realizado nas universidades, 70,6\% dos alunos no Rio de Janeiro e 46,9\% dos alunos em São Paulo haviam adquirido dentes para as suas atividades acadêmicas em 2001. Ainda no mesmo estudo os resultados indicam que a maior parte das encomendas de dentes foi feita em cemitérios por intermédio de coveiros, indicando que este 'crime' vem constantemente sendo cometido". ${ }^{20}$

Em outro estudo ${ }^{5}$, constatou-se "uma grande resistência por parte dos alunos em doarem suas coleções particulares de dentes, muitas vezes provenientes de pais dentistas que armazenaram o material ao longo dos anos, bem como de outros profissionais, colegas do curso em estágios mais avançados e/ou 
egressos", o que dificulta a criação dos BDH nos cursos de Odontologia.

Nos casos de pesquisa, os pesquisadores devem solicitar ao BDH da sua instituição e encaminhar com documento anexo para o CEP. "Atualmente os Comitês de Ética em Pesquisa (CEP) não aprovam pesquisas que utilizam dentes humanos cuja origem não seja comprovada ou legalizada". ${ }^{13}$

Os autores ${ }^{7,8,14,15,18,20}$ reconhecem que os pesquisadores e acadêmicos dos curso de Odontologia, têm a obrigação do conhecimento da Lei $\mathrm{n}^{\circ} 9.434$ de 04/02/1997, que dispõe sobre a remoção de órgãos, tecidos e partes do corpo humano para fins de transplante e tratamento e de outras procedências. "Os estudantes, ao comprarem dentes, podem ser enquadrados nas leis penais e/ou civis, mesmo alegando não saberem que o ato é crime, pois o artigo $3^{\circ}$ do Código Civil diz que 'ninguém se escusa de cumprir a lei, alegando que não a conhece'”. ${ }^{16}$

"A inexistência de bancos de dentes vinculados às instituições de ensino ferem princípios éticos e legais, pois muitas vezes os alunos utilizam dentes de origem duvidosa, como cemitérios; e por outro lado incentiva a comercialização ilegal de dentes, pois os acadêmicos que não conseguem obter dentes para utilizarem em suas aulas práticas são obrigados a compra-los para não serem prejudicados em suas atividades. Este cenário tem também implicações de biossegurança, pois muitas vezes os dentes não são acondicionados de forma adequada e podem ser veículo para transmissão de doenças". ${ }^{16}$

O que incentiva a comercialização e troca das unidades dentárias, uma vez que a sua utilização é exigência nos cursos e faz com que os discentes busquem adotar meios ilícitos e não éticos para a sua aquisição.

Os Bancos de Dentes Humanos ainda não participam da rotina de muitos cursos de graduação em Odontologia no Brasil, o que se constata na pesquisa sobre "Levantamento dos Bancos de Dentes Humanos dos Cursos de Odontologia do Brasil e Experiência na criação do Banco de Dentes Humanos da Universidade Estadual de Feira de Santana, Bahia” ${ }^{25}$ em 2011, que dos 196 cursos de Odontologia, apenas 64 cursos apresentam $\mathrm{BDH}$, conforme mapeamento realizado nesta pesquisa de doutorado com ênfase em Bioética.

Portanto, a criação dos BDH nos cursos de Odontologia é exigida a fim de legalizar a utilização das unidades dentárias na prática acadêmica e na pesquisa científica, assim com direcionar e facilitar a captação do elemento dental, com a normatização e a organização com base nas leis vigentes no País, além dos cuidados com a Biossegurança.

Por outro lado, não se pode esquecer que o dente é um material biológico humano e como tal está inserido na Resolução CNS n ${ }^{\circ} 441$ de 12/05/2011 que regulamenta os Biobancos e os Biorepositórios com normas específicas onde os "Biobancos são fundamentais para a pesquisa, contribuindo também para as atividades de assistência clínica, pois permitem o armazenamento de amostras biológicas - como sangue, cordão umbilical, tecidos tumorais e normais, entre outras - associadas aos dados clínicos dos pacientes. Um exemplo na pesquisa é o estudo de biomarcadores, características que podem ser mensuradas e que podem indicar processos biológicos e patológicos, possuindo valores de previsão e de prognóstico. Já nas atividades assistenciais, registra-se a importância dos bancos de tumores, que contribuem para o diagnóstico e acompanhamento do estadiamento tumoral". ${ }^{26}$

Diante desta definição dos Biobancos se concluem que, houve uma mudança na condição do elemento dental de instrumento básico para o ensino acadêmico nos cursos de graduação e pós-graduação de Odontologia para material orgânico humano monitorado nas atividades de pesquisa, contribuindo assim nas atividades de assistência clínica.

Sugere-se a criação de uma lei específica para os Bancos de Dentes Humanos no Brasil, o qual contemple principalmente o Ensino e a Extensão, uma vez que a Pesquisa esta inserida na Resolução CNS n ${ }^{\circ}$ 441 de 12/05/2011 e desta forma inibir a aquisição ilegal de dentes humanos.

Espera-se que esta revisão de literatura ainda que incipiente, desperte nos leitores o interesse pelo assunto, estimule novas pesquisas e reforce a importância da criação dos $\mathrm{BDH}$ nos cursos de Odontologia como órgão institucional que auxilia o ensino da Odontologia, e contribui para o avanço da pesquisa na área da saúde em geral.

\section{ABSTRACT}

\section{Human tooth bank in Brazil: a review of literature}

This article describes the results of a literature review on Brazil's "Human Tooth Banks (HTB)," conducted to gain more knowledge into the scientific literature available on the country's HTBs, their relationship with teaching, research and extension, as well as their administrative structure and legal implications, based on bioethics. The study was based on data from the Virtual Dental Health Library and specialized books. Eighty-one articles and two books 
were retrieved, from which twenty-three articles and two books were selected, one on Human Tooth Banks and the other on ANVISA (Brazilian Health Surveillance Agency) Dental Services. The analyzed texts were classified into three categories: Education/Research, Organization/Structure of HTBs and Bioethics/Biosafety. We concluded that few HTB-related studies were conducted in Brazilian dentistry courses, thus revealing a need for new reflections in dentistry as concerns bioethics in creating an HTB.

\section{DESCRIPTORS}

Tooth Bank. Biosafety. Bioethics. Dentistry Courses.

\section{REFERÊNCIAS}

1. Brasil. MS. Manual de prevenção e controle de riscos em serviços odontológicos. Brasília; Ministério da Saúde, 2006;107-111.

2. Lubisco NML, Vieira SC, Santana IV. Manual de estilo acadêmico- Monografias, Dissertações e Teses- Salvador: EDUFBA, 2008. 145p.

3. Vanzelli M, Imparato JCP. Banco de Dentes: uma idéia promissora. Stomatos, RGS: Canoas, 2003; 9 (16): 59-60 jan/jun.

4. Imparato JCP. et col. Banco de Dentes Humanos. $1^{\mathrm{a}}$ ed. Paraná: Editora Maio, 2003.

5. Zucco D, Kobe R, Fabre C, Madeira L, Baratto Filho, F. Avaliação do nível de conhecimento dos acadêmicos do curso de Odontologia da UNIVILLE sobre a utilização de dentes extraídos na graduação e banco de dentes. RSBO, 2006 ; 3 (1):5458 ISSN 1806-7727

6. Pires LAG, Cerveira J. A Bioética na Odontologia. Stomatos. 2003; 9 (17):7-12.

7. Costa SM, Mameluque S, Brandão EL, Melo AEMA, Pires CPAB, Rezende EJC, Alves KM. Dentes humanos no ensino odontológico: procedência, utilização, descontaminação e armazenamento pelos acadêmicos da UNIMONTES. Revista da ABENO. 2005; 7 (1):6-12.

8. Freitas ABDA, Castro CDL, Sett GS, Barros LM, Moreira A N, Magalhães CS. Uso de dentes extraídos nas pesquisas odontológicas publicadas em periódicos Brasileiros de acesso online gratuito: um estudo sob o prisma da bioética. Arquivos em Odontologia. 2010; 46 (3):136-143.

9. Muratori G. La Banca del Dente. Dental Cadmos.1969.

10. Mathieu L, Fleurette J, Transy MJ .Les Transplantions Dentaires: constitution d'une Banque de dents et problèmes de stérilisation. Ann Odontostomatol (Lyon) 1970.

11. Gabrielli F, Dinelli W, Fontana UF, Porto, CLA RGO, Porto Alegre.1981;29(2):83-87.

12. Vinholes J I A, Fernandes D C, Ritzel IF. Banco de Dentes Humanos no curso de Odontologia da ULBRA- Campus Torres. Conversas Interdisciplinares 1(1) ISSN 2176-1051.
13. Moreira L, Genari B, Stello R, Collares FM, Samuel SMW. Banco de Dentes Humanos para o Ensino e Pesquisa Odontológica. Rev. Fac. Odontol. Porto Alegre. 2009; 50 (1):34-37.

14. Ferreira EL, Fariniuk LF, Cavali AÉC, Baratto Filho F, Ambrósio AR. Banco de dentes: ética e legalidade no ensino, pesquisa e tratamento odontológico. RBO. 2003; 60 (2):120-122,mar/abr.

15. Marin E, Zorzin D, Mainardi AP, Oliveira MDM. Estruturação do Banco de dentes Humanos Decíduos da Universidade Federal de Santa Maria/ RS/ Brasil. Passo Fundo. 2005; 10 (2):7-9.

16. Pinto SL, Silva SP, Barros LM, Tavares EP, Silva JBOR, Freitas ABDA. Conhecimento Popular, Acadêmico e Profissional sobre Banco de Dentes Humanos. Pesq Bras Odontoped Clin Integr, João Pessoa, 2009; 9 (1):101-106, jan/abr.

17. Garbin C A S, Garbin AJI, Santos KTS, Pacheco, AC. Percepção de acadêmicos de odontologia sobre clonagem, doação de órgãos e Banco de Dentes. RPG. 2008 ; 15 (4):225-60.

18. Nassif A, Tieri F, Ana P, Botta S, Imparato JCP. Estruturação de um Banco de Dentes Humanos. Pesq Odontol Bras. 2003;17(Supl 1): 70-4.

19. Begosso M, Imparato JCP, Duarte D. Estagio atual da organização dos bancos de dentes humanos nas faculdades de Odontologia do território brasileiro. RPG. 2001;8 (1):23-28.

20. Paula S, Bittencourt LP, Pimentel E, Gabrielli Filho PA, Imparato JCP. Comercialização de dentes nas Universidades. Pesq Bras Odontoped Clin Integr. 2001; 1(3) set/dez. 38-41.

21. Maggioni A R, Maggioni AR, Scelza MFZ, Silva LE, Salgado VE, Borges DO, Maciel ACC Banco de dentes Humanos na percepção dos acadêmicos da Faculdade de Odontologia da Universidade Federal Fluminense. Rev.Fluminense de Odontol. Rio de Janeiro. 2010; 33 (1):27-30.

22. Poletto MM, Moreira M, Dias MM, Lopes MGK, Lavoranti OJ, Pizzatto E. Banco de dentes humanos: perfil sociocultural de um grupo de doadores. RGO, Porto Alegre, 2010; 58(1): 91$94 \mathrm{jan} / \mathrm{mar}$.

23. Vanzelli M, Ramos DLP, Imparato JCP. Valorização do Dente como um órgão. In: Banco de dentes Humanos. Paraná: Editora Maio; 2003.p35-37.

24. Silva M. Compêndio de Odontologia Legal. Rio de Janeiro: Medsi,1997 p.89-90.

25. Pereira DQ. Levantamento dos Bancos de Dentes Humanos dos Cursos de Odontologia do Brasil e a Experiência na criação do Banco de Dentes Humanos da Universidade Estadual de Feira de Santana - Bahia. [tese]. Salvador (BA): Programa de Pós-graduação de Medicina e Saúde, Universidade Federal da Bahia; 2012.

26. Informes Técnicos Institucionais. Diretrizes nacionais para biorrepositórios e Biobancos de materiais humanos em pesquisa. Ministério da Saúde. Rev.Saúde Pública, 2009; 43(5): 898-9.

Recebido em 08/10/2012

Aceito em 10/12/2012 